\title{
Research and Realization of a Master-Slave Robotic System for Retinal Vascular Bypass Surgery
}

Chang-Yan He ${ }^{1}$, Long Huang ${ }^{1}$, Yang Yang ${ }^{1 *} \mathbb{D}$, Qing-Feng Liang ${ }^{2}$ and Yong-Kang Li ${ }^{1}$

\begin{abstract}
Retinal surgery continues to be one of the most technical demanding surgeries for its high manipulation accuracy requirement, small and constrained workspace, and delicate retinal tissue. Robotic systems have the potential to enhance and expand the capabilities of surgeons during retinal surgery. Thus, focusing on retinal vessel bypass surgery, a master-slave robot system is developed in this paper. This robotic system is designed based on characteristics of retinal vascular bypass surgery and analysis of the surgical workspace in eyeball. A novel end-effector of two degrees of freedom is designed and a novel remote center of motion mechanism is adopted in the robot structure. The kinematics and the mapping relationship are then established, the gravity compensation control strategy and the hand tremor elimination algorithm are applied to achieve the high motion accuracy. The experiments on an artificial eyeball and an in vitro porcine eye are conducted, verifying the feasibility of this system.
\end{abstract}

Keywords: Retinal robotic system, Mechanism design, Kinematics, Gravity compensation

\section{Introduction}

Retinal vein occlusion (RVO), as the main cause of blindness in retinal vascular disease after diabetic retinopathy [1], has affected estimated 16.4 million adults worldwide [2]. It is caused by the formation of a clot blocking the circulation of blood in retinal veins. With blockage, pressure increases in capillaries, causing blood leak, macula obscurity and other various retinopathies, and ultimately loss of sight. So far, the most effective way to treat this disease is to apply intraocular surgery, such as retinal vascular bypass surgery (RVBS). RVBS is a new and promising treatment. However, manual RVBS is difficult and risky because of two problems: the limited workspace and the rigorous requirements of manipulating accuracy. First, the target retinal tissue is as thin as $25 \mu \mathrm{m}$ and the retinal vessel diameter is as tiny as $80 \mu \mathrm{m}$, while surgeon physiological hand tremor's amplitude is $182 \mu \mathrm{m}$ [3]. Thus, eye tissue will suffer serious injuries from any

\footnotetext{
*Correspondence: yang_mech@126.com

${ }^{1}$ School of Mechanical Engineering and Automation, Beihang University, Beijing 100191, China

Full list of author information is available at the end of the article
}

surgoen's unintended movement. Secondly, surgeon has to manipulate the instruments in a confined space under microscope, all the surgical operations need to be conducted through a trocar mounted on one side of sclera (the white part of eyeball). Therefore, the surgery result is subjected to the surgeon's limited dexterity.

To overcome the two difficulties mentioned above, scholars have resorted to the microsurgical robot systems with high motion accuracy and stability. A fair number of research has been carried out unremittingly to pursue the technical breakthroughs in this field [4]. One of the first ocular robotic systems is the stereotaxical micro- manipulator (SMOS) developed by Guerrouad and Vidal in 1989. SMOS can achieve six degrees of freedom (DoF) motion using a spherical micromanipulator [5]. A similar robotic system was introduced by $\mathrm{Yu}$ et al. [6] for intravascular drug delivery with a spherical manipulator. Charles et al. [7] described a master-slave robotic system called RAMS for eye surgery consisting of a six DoF cable-driven manipulator with 10-micron precision in 1997. Wei et al. [8] developed a novel hybrid two-armed microsurgical robot, and each arm consists of a two DoF intraocular dexterity manipulator and a 
six DoF parallel stage. Ueta et al. [9] at Tokyo University proposed a spherical manipulator to assist vitreoretinal surgery in 2009. Taylor's group in John Hopkins University (JHU) [10] has researched on steady hand eye robot (SHER) for years. SHER is a co-operative surgical device, which allows surgeon directly hold the robot end-effector and manipulate the instrument. Rahimy et al. [11] developed IRISS eye robot to perform cataract surgery, IRISS has two circle sliders to construct manipulators.

Recently, Nasseri et al. [12] introduced a very compact robotic system for ophthalmic surgery as small as the average adult hand in 2013. Pooerten's group in Katholieke Universiteit Leuven [13] developed a comanipulation and tele-manipulation robotic system for retinal surgery composed of a salve manipulator and a cable-driven master controller. Researchers from TU Eindhoven [14] conducted the similar research with Pooerten's and proposed the PRESEYES micromanipulator. PRESEYES consists of a motion controller and a table mounted manipulator. Yang et al. in Beihang University have researched on the robotic system for eye surgery for years, proposed corneal hyper-viscoelastic model [15], investigated needle insertion force in robot assisted corneal suturing $[16,17]$, and developed a robot assistance for retinal surgery [18]. Researchers also investigated the application of da Vinci surgical system in eye surgery [19], however, due to its cumbersome end-effector, da Vinci system is not suitable to conduct eye surgery. Besides the robotic manipulators, other types of robotic devices have been developed, including handheld devices [20, 21], flexible micromanipulators [22, 23], untethered micro robots [24], force-sensing micro-instruments [25, 26]. Recently, two robot assisted retinal surgeries have been performed successfully on human patients [27, 28], demonstrating the clinical feasibility of the robotic technology for retinal microsurgery.

A series of problems about robot assisted retinal surgery have been solved in the past years. However, little research has investigated robotic assisted RVBS in the previous works, and further studies are needed to address the motion error compensation and the tremor canceling in the microsurgical robotic systems. Therefore, this work proposed a master-slave robotic system aimed to enhance the robot motion accuracy and reduce the requirements of surgeon's operation skills in RVBS. In the rest of this paper, the surgical workspace in the eyeball was first analyzed on the basis of the procedures of RVBS. Secondly, the robotic system design and the structure of a novel end-effector was presented. Then, the kinematics and the coordinates mapping relationship of the robotic system were established, and the gravity compensation control strategy was applied to achieve high robotic system motion accuracy. Next, the recursive least square algorithm was adopted to eliminate the hand tremors from the master manipulators. Finally, the experiments conducted an artificial eyeball model and a porcine eyeball demonstrated the feasibility of the robotic system.

\section{Retinal Vascular Bypass Surgery (RVBS)}

Today, no treatment has been proven effective for RVO [29], the current solutions including pharmaceutical method (e.g. retinal vein cannulation) and laser surgery cannot permanently solve the retinal ischemia. However, RVBS has been widely recognized as a promising treatment for vascular occlusion due to its excellent treatment effect in recent years. The Eye Hospital of Wenzhou Medical College has accomplished the first RVBS on a vivo porcine model in 2009, successfully cured the RVO preset manually in the porcine eyeball [30].

The main operation procedures of the RVBS can be concluded as follows [31]:

(i) extract the crystalline lens and drill two or three operative incisions on sclera to fix trocars as the access to insert the surgical instruments;

(ii) import the tiny vascular prosthesis use a hollow needle through the trocar into eyeball, and then clamping the vascular prosthesis by an intraocular microforceps inserted through another trocar;

(iii) operate bypass for the blocked blood vessel by inserting two ends of the vascular prosthesis into two sides of blockage;

(iv) operate the end-diathermy.

\section{Robotic System Design}

\subsection{Instruments Workspace}

The adult human eyeball can be considered as a revolution around the optical axis with an endocoele of $21 \mathrm{~mm}$ diameter approximately, and the region where retinal diseases take place commonly can be regarded as a spherical cap of $18.2 \mathrm{~mm}$ diameter at the bottom of the eyeball. The radius angle of the arc generatrix on this spherical cap is calculated as $41^{\circ}$, this value is modified as $45^{\circ}$ in this paper to fully cover the workspace. The new spherical cap is nominated as focus range. To meet requirements of the surgical space and to avoid interference of the instruments, the angle between two planes passing the optical axis and the incisions on sclera is set as $120^{\circ}$. The motion range of instrument is determined as shown in Figure 1, and the incision points for the surgical instrument are solved as $P_{1}(6.55,-3.782,7.283)$ and $P_{2}(-6.55$, $-3.782,7.283)$ in the coordinate system $\{E Y E\}$ whose origin located on the spherical center of the eyeball. The calculated instrument motion range is used as the design parameters for the robot mechanism. 


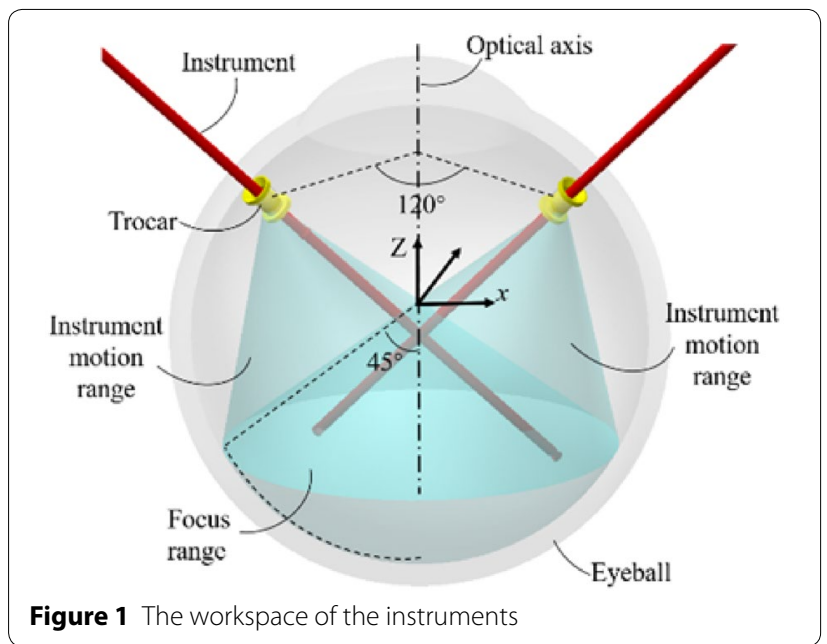

\subsection{Remote Center of Motion (RCM) Manipulator Design}

According to the description of RVBS, the surgical instruments are inserted through sclera incisions into eyeball to conduct surgical tasks. To ensure that instruments does not tear the incisions, a remote center of motion (RCM) mechanism is designed and applied in the robotic manipulators. RCM mechanism can provide a virtual pivot for the manipulator without having a physically constrained fulcrum point, and the virtual pivot corresponds to the incision on the patient's sclera. In our previous work [32], a novel RCM mechanism based on the double parallelogram mechanism is proposed, and its construction process is shown as Figure 2. This new RCM mechanism can provide a 3-DoF motion consisting of a 2-DoF tilting motion and an additional tool-insertion motion. Notably, the tool insertion motion is achieved by the coupled movements of joints on the base. Thus, no actuators need to be loaded on the end of manipulator, which means stiffness of the mechanism could be improved. Based on this novel RCM mechanism, a RCM manipulator is designed and developed as shown in Figure 3. The rotation platform (MRS102, BOCIC, Co., Ltd, China) is applied to provide 1-DoF tilting motion, and the combination of a DC motor (35YF22GN, Times Brilliant, Co., Ltd, China) and a stepper motor (28BYG250, KDE, Co., Ltd, China) is used to provide 1-DoF tilting motion and the tool-insertion motion.

\subsection{End-effector Design}

Two slave manipulators have different end-effectors to conduct different surgery tasks. The left manipulator is equipped with a hollow needle or an intraocular cautery or a vitreous cutter in different steps, and the right manipulator is equipped with the intraocular microforceps. Unlike the instruments of the left end-effector, the intraocular microforceps needs to rotate around its own axis, and its jaw needs to be opened and closed to perform surgical tasks. Thus two DoF (rotational DoF and forceps open/close DoF) are required in right end-effector.

To solve the aforementioned problems, a novel end-effector is developed as shown in Figure 4. The

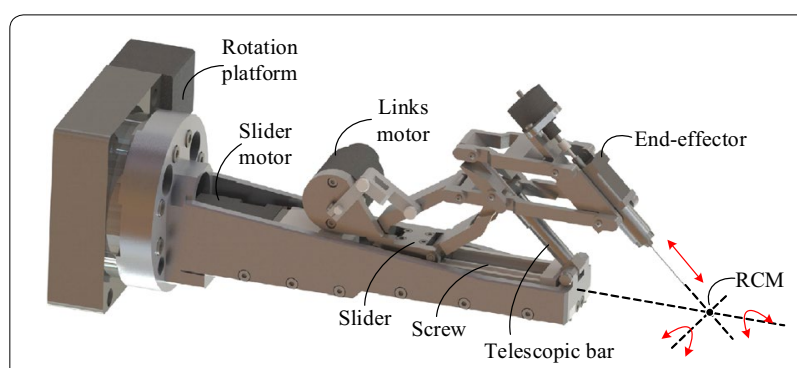

Figure 3 Structure of the RCM manipulator

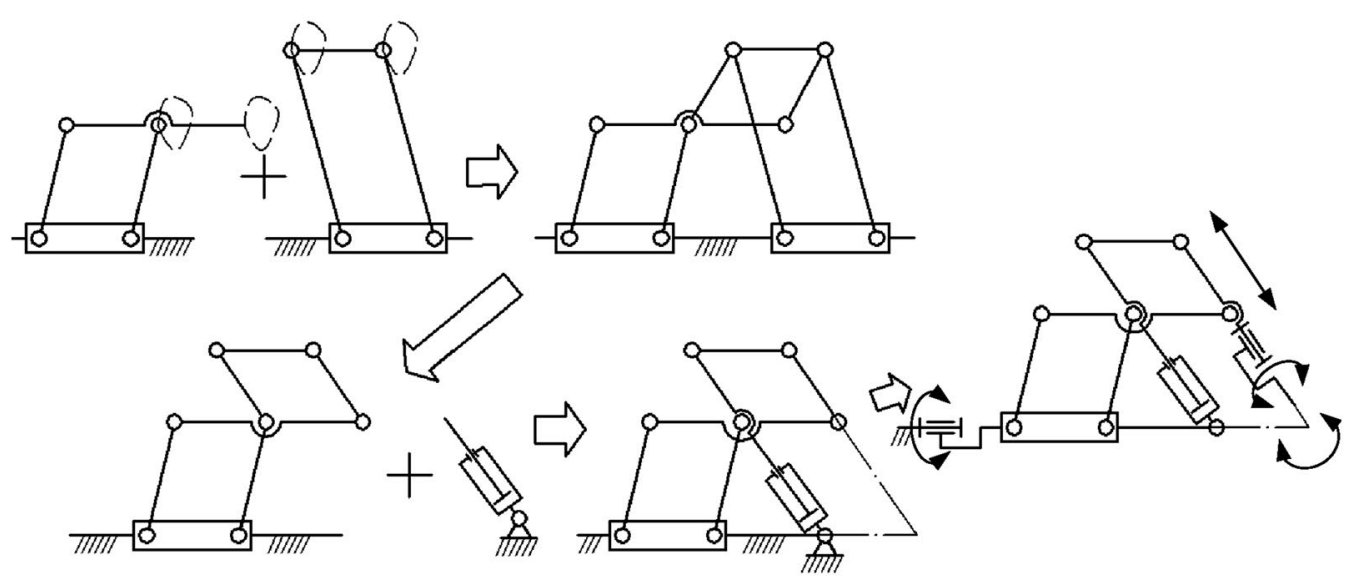

Figure 2 Construction process of the RCM mechanism 


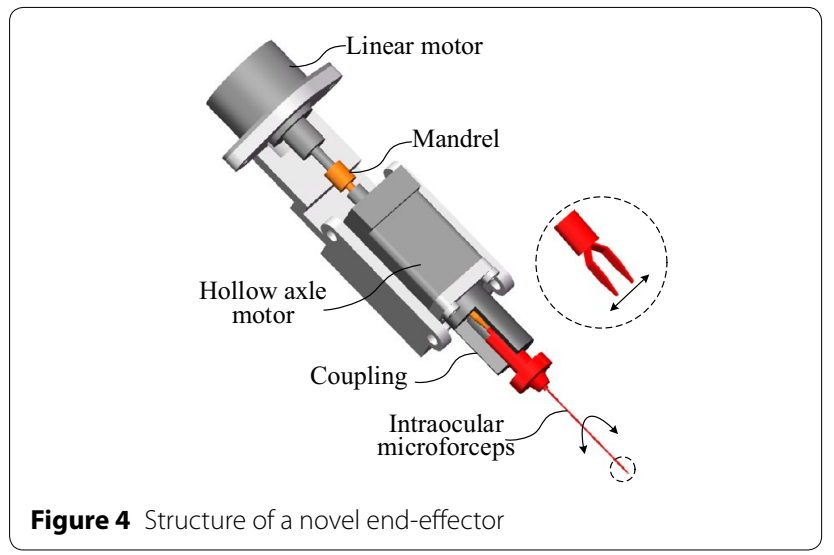

end-effector has two DoF enabling the microforceps to rotate and clamp. Based on the unique mechanical structure of intraocular microforceps (Synergetics, O'Fallon, MO), a linear motor (25BYZ-A013, BTMotor Co., Ltd, China) is used to achieve opening and closing of microforceps. Meanwhile, to make the endeffector structure more compact, a hollow axle motor (SM20BYG30, Smartever Co., Ltd, China) is applied to rotate the microforceps. One mandrel is inserted through the hollow axle motor. One end of the mandrel is fixed with the output axis of a linear motor, and the other end clings to open/close trigger of the microforceps. Also, the microforceps is coupled with the output axis of the hollow axle motor. With this novel endeffector, the open size and the direction of the microforceps can be controlled so that objects of different size can be grasped.

\subsection{Robotic System Implementation}

Based on the surgery procedures of RVBS, the prototype of a master-slave surgical robotic system has been developed and realized as shown in Figure 5, and the structure of the robotic system is presented in Figure 6. The robotic system consists of the following parts:

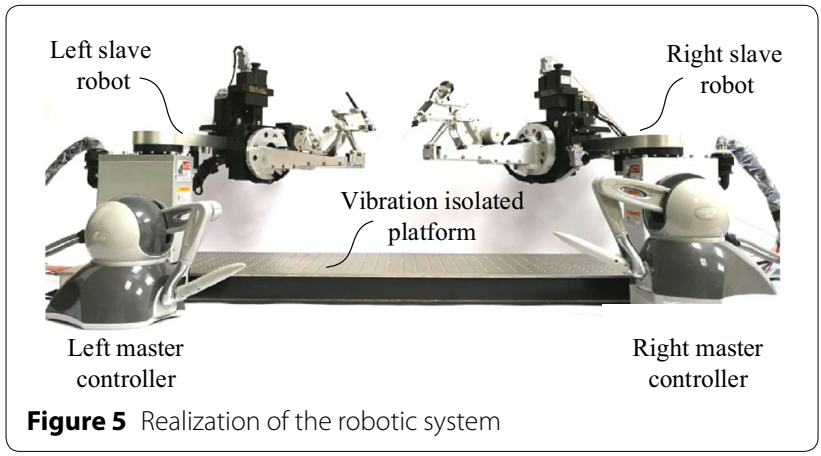

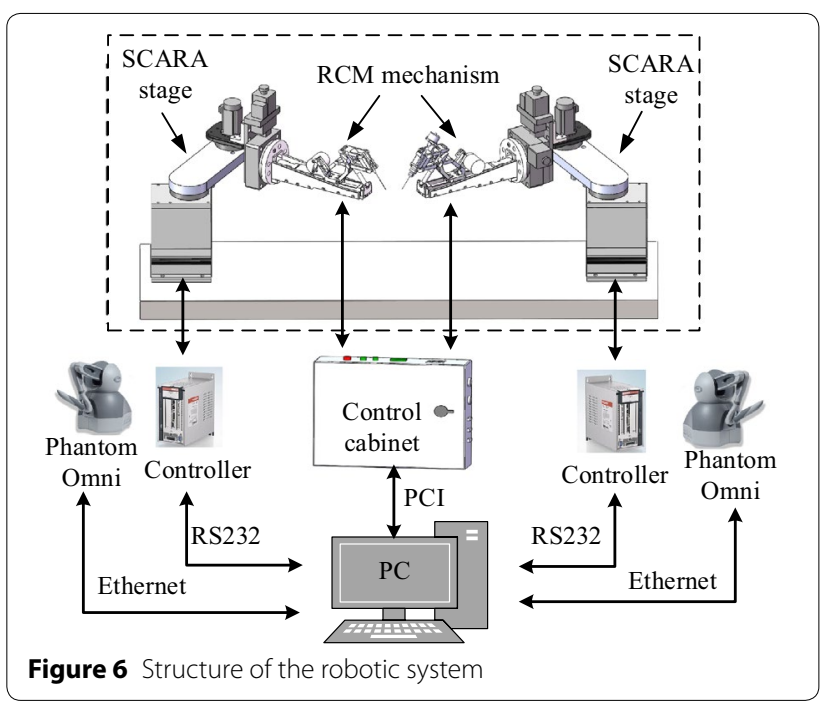

- two PHANTOM Omnis with six DoF (SensAble Technologies Inc.) served as the master controllers;

- two identical slave manipulators made up of the SCARA stages of three DoF (Yamaha Motor Co.) and the novel RCM mechanisms;

- a novel end-effector of two DoF;

- a PC host computer and a custom robot control cabinet consisting of a Programmable Multi-Axis Controller (PMAC) (Googol Technology (HK) Ltd.).

The SCARA stage is used to adjust the endpoint position of the slave manipulator at the beginning of the surgery, and the RCM mechanism is responsible for the intraocular operations.

\section{System Controller Design}

\subsection{Controller Implementation}

The robotic control subsystem is made up of off the shelf and custom-made electronics as shown in Figure 7. It includes four main components: PC host computer, master controller, control cabinet and slave manipulator. It should be noted that both the PC human machine interface (HMI) operated by the surgeon assistant and the master controller operated by the main surgeon have the access to the slave manipulator motion control, but there are two differences between these two motion commanders:

Firstly, the HMI has a higher priority than the master controller, and the instructions from the latter can be blocked by the commands from former in any time to avoid the manipulation errors from the main surgeon, such as accidental hand movements.

Secondly, motor commands from the HMI are valid during whole surgical process, while the main surgeon 


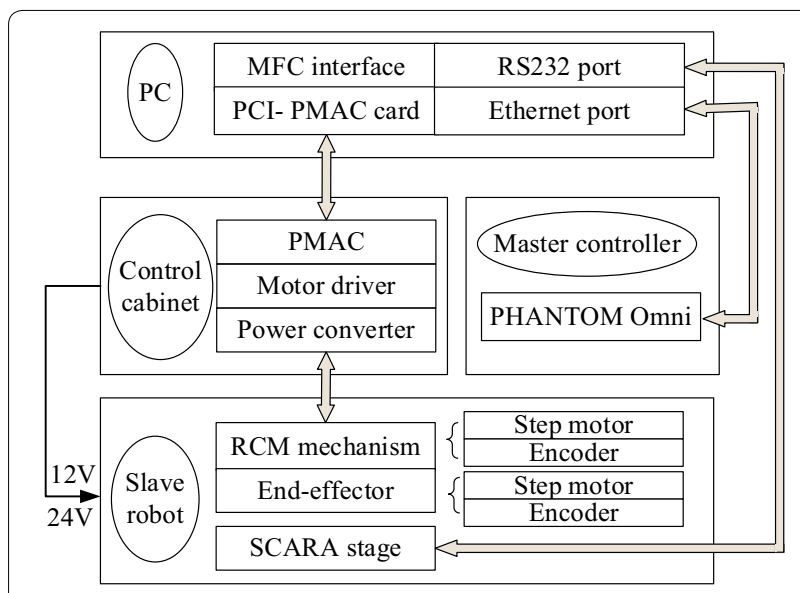

Figure 7 Control subsystem schematics

control mode is active only during the intraocular operation procedure.

The current implementation of control system is shown in Figure 8. The position increment $X_{m}$ of the master controller is calculated using master kinematics and tremor cancelling algorithm in $\mathrm{PC}$, and the slave manipulator motion increment $X_{s}$ is obtained by the inverse-kinematics and the gravity compensation strategy. In addition, the loop control of the robotic system is accomplished by the surgeon visual feedback with a microscope. To improve the surgeon operating accuracy, the surgeon manipulation space is enlarged $K$ times compared with the real eyeball space.

\subsection{Kinematics Analysis}

The kinematics results of the master controller can be directly obtained from the PHANTOM Omni calculation program, thus only the kinematics of the slave manipulators are analyzed in this work. The kinematics model for

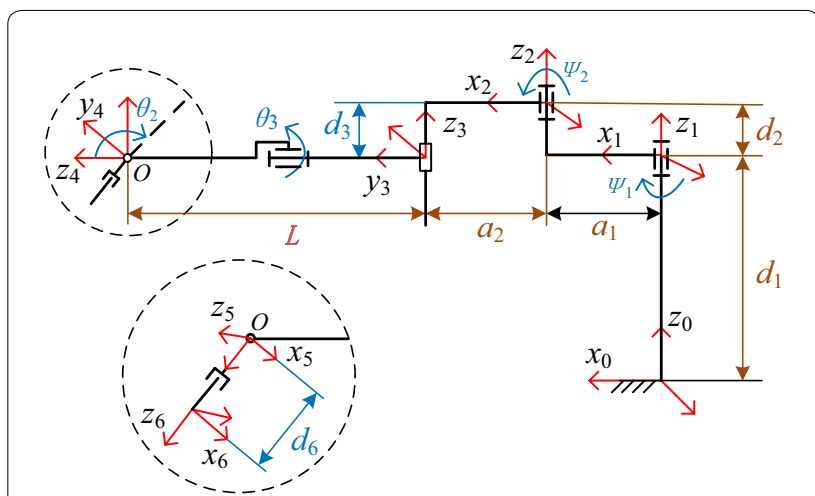

Figure 9 Link-pole coordinate system of one slave manipulator

one slave manipulator is established under link-pole coordinate system with the method of Denavit-Hartenberg [33] as shown in Figure 9, where $\Psi_{1}, \Psi_{2}, d_{3}, \theta_{2}, \theta_{3}$, and $d_{6}$ are the joint variables, $a_{1}, a_{2}, d_{1}, d_{2}$, and $L$ are the geometry parameters, $\left\{O_{0} x_{0} y_{0} z_{0}\right\}$ is the base coordinate system, $\left\{O_{i} x_{i} y_{i} z_{i}\right\}, i=1,2, \ldots, 6$ is the coordinate system for each joint. It should be noted that joint 5 represents the RCM mechanism, since the RCM can be considered as one revolution joint around the point $O$. The RCM mechanism is shown in Figure 10, where $O$ is the fixed point of instrument motion, $l_{1}, l_{2}$, and $L$ are the geometry parameters, $\theta_{1}$, $\theta_{2}$, and $\theta_{3}$ are the drive joint variables, and $x_{1}$ is the passive variable. The kinematics of the first three joints and the last three joints are solved separately since the two sets of joints are decoupled from each other. Equation (1) and Eq. (3) show the forward kinematic solutions for first three joints and the last three joints respectively, $P_{O}$ in Eq. (2) represents the coordinate of the fixed point of the RCM mechanism in the coordinate system $\left\{O x_{0} y_{0} z_{0}\right\}$. Equation (4) and Eq. (5) show the inverse kinematic solutions for the two sets of joints, respectively.

$$
\begin{aligned}
& { }_{0}^{3} T={ }_{0}^{1} T_{1}^{2} T_{2}^{3} T= \\
& {\left[\begin{array}{lll}
\sin \left(\psi_{1}+\psi_{2}\right) & \cos \left(\psi_{1}+\psi_{2}\right) & 0 a_{1} \cos \psi_{1}+a_{2} \cos \left(\psi_{1}+\psi_{2}\right) \\
-\cos \left(\psi_{1}+\psi_{2}\right) & \sin \left(\psi_{1}+\psi_{2}\right) & 0 a_{1} \sin \psi_{1}+a_{2} \sin \left(\psi_{1}+\psi_{2}\right) \\
0 & 0 & 1 d_{1}+d_{2}+d_{3} \\
0 & 0 & 01
\end{array}\right],}
\end{aligned}
$$

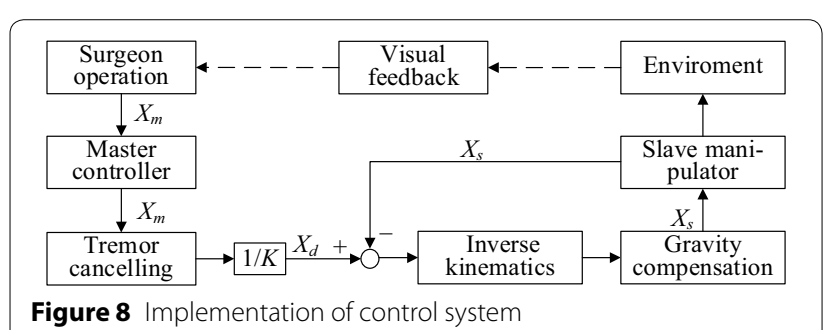

$$
P_{O}={ }_{0}^{3} T \times\left[\begin{array}{c}
0 \\
L \\
0 \\
1
\end{array}\right]=\left[\begin{array}{c}
a_{1} \cos \psi_{1}+\left(a_{2}+L\right) \cos \left(\psi_{1}+\psi_{2}\right) \\
a_{1} \sin \psi_{1}+\left(a_{2}+L\right) \sin \left(\psi_{1}+\psi_{2}\right) \\
d_{1}+d_{2}+d_{3}
\end{array}\right]
$$

Figure 8 Implementation of control system 


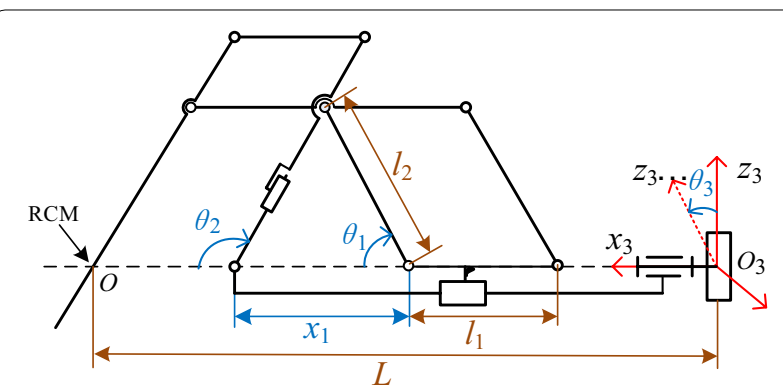

Figure 10 Structure sketch of the RCM mechanism

$$
\begin{aligned}
& { }_{3}^{6} T={ }_{3}^{4} T_{4}^{5} T_{5}^{6} T= \\
& {\left[\begin{array}{cccc}
-\cos \theta_{2} \sin \theta_{3} & -\cos \theta_{3} & -\sin \theta_{3} \sin \theta_{2} & -d_{6} \sin \theta_{3} \sin \theta_{2} \\
-\sin \theta_{2} & 0 & -\cos \theta_{2} & L-\cos \theta_{2} d_{6} \\
\cos \theta_{3} \cos \theta_{2} & \sin \theta_{3} & -\cos \theta_{3} \sin \theta_{2} & -d_{6} \cos \theta_{3} \sin \theta_{2} \\
0 & 0 & 0 & 1
\end{array}\right],}
\end{aligned}
$$

$$
\left\{\begin{array}{l}
\psi_{1}=\arctan \frac{\left(a_{1}+\left(a_{2}+L\right) \cos \psi_{2}\right) P_{o y}-\left(a_{2}+L\right) \sin \psi_{2} P_{o x}}{\left(a_{1}+\left(a_{2}+L\right) \cos \psi_{2}\right) P_{o x}+\left(a_{2}+L\right) \sin \psi_{2} P_{o y}} \\
\psi_{2}=\cos ^{-1} \frac{P_{x}^{2}+P_{y}^{2}-a_{1}^{2}-\left(a_{2}+L\right)^{2}}{2 a_{1}\left(a_{2}+L\right)} \\
d_{2}=P_{z}-d_{0}-d_{1}
\end{array}\right.
$$

$$
\left\{\begin{array}{l}
\theta_{1}=\arctan \frac{2 l_{2} x_{1} \sin \theta_{2}}{x_{1}^{2}+l_{1}^{2}-l_{2}^{2}}, \\
\theta_{2}=\arctan \frac{P_{e x}}{\sin \theta_{3}\left(P_{e y}-L\right)}, \\
\theta_{3}=\arctan \frac{P_{e x}}{P_{e y}}, \\
x_{1}=-l_{2} \cos \theta_{2} \pm \sqrt{l_{2}^{2} \cos ^{2} \theta_{2}-l_{2}^{2}+l_{1}^{2}},
\end{array}\right.
$$

where $\left\{P_{o x}, P_{o y}, P_{o z}\right\}$ is coordinate of the fixed point of RCM mechanism. $\left\{P_{e x}, P_{e y}, P_{e z}\right\}$ is coordinate of the endeffector end point.

\subsection{Gravity Compensation}

Because the RCM mechanism comprises of multiple links, the gravity of these components will apply extra force for the joints and affect the location accuracy of instrument. To achieve fine control of the RCM mechanism, the gravity compensation for actuator control is solved via dynamic analysis and the computed torque method [34].

Firstly, the equivalent model of the RCM mechanism is established by removing the redundant constraints as shown in Figure 11, where $O$ is the motion fixed point, $s_{i}$ is the norm of radius vector for the link $i, \phi_{i}$ is the direction of the radius vector $i, \theta_{1}, \theta_{2}$, and $\theta_{3}$ are drive

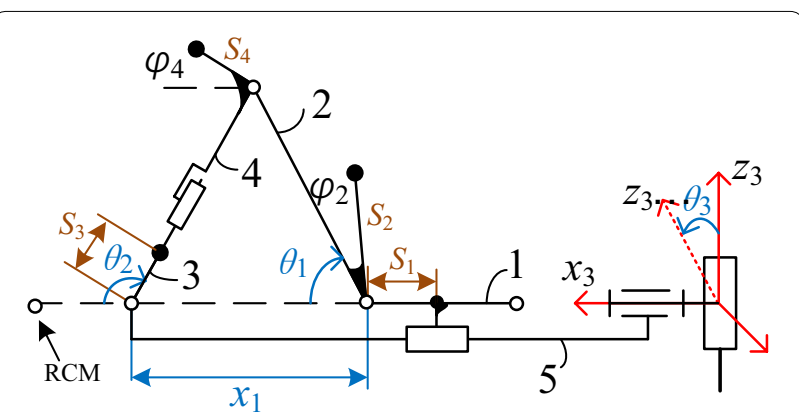

Figure 11 RCM mechanism is equivalent to 5 links

joint variables, $x_{1}$ is passive variable, and $\left\{O^{\prime} x^{\prime} y^{\prime} z^{\prime}\right\}$ is the base coordinate system of RCM mechanism. The centroid radius vectors of links can be acquired by Eq. (6). Setting the variables $\theta_{1}, \theta_{2}, \theta_{3}$ and $x_{1}$ as the generalized coordinates, angular velocity and centroid velocity of equivalent links can be acquired by Eq. (7). Then based on Eq. (6) and Eq. (7), the Lagrange equation is adopted to establish the dynamic model as shown in Eq. (8). Finally, the gravity compensation controller is designed based on the dynamic model and proportion-derivative control algorithm.

$$
\boldsymbol{r}_{i}=\frac{\sum_{j=1}^{n_{i}} m_{j} \boldsymbol{r}_{j}}{\sum_{j=1}^{n_{i}} m_{j}}
$$

where $n_{i}$ is the number of actual links corresponding to the equivalent link $i, \boldsymbol{r}_{j}$ is the centroid radius vector of the link $j, m_{j}$ is the mass of link $j$.

$$
\left\{\begin{array}{c}
\dot{\boldsymbol{\Phi}}_{\boldsymbol{i}}=\frac{\partial \boldsymbol{\Phi}_{\boldsymbol{i}}}{\partial x_{1}} \dot{x}_{1}+\frac{\partial \boldsymbol{\Phi}_{\boldsymbol{i}}}{\partial \theta_{1}} \dot{\theta}_{1}+\frac{\partial \boldsymbol{\Phi}_{\boldsymbol{i}}}{\partial \theta_{2}} \dot{\theta}_{2}+\frac{\partial \boldsymbol{\Phi}_{\boldsymbol{i}}}{\partial \theta_{3}} \dot{\theta}_{3}, \\
\dot{x}_{S i}=\frac{\partial x_{S i}}{\partial x_{1}} \dot{x}_{1}+\frac{\partial x_{S i}}{\partial \theta_{1}} \dot{\theta}_{1}+\frac{\partial x_{S i}}{\partial \theta_{2}} \dot{\theta}_{2}+\frac{\partial x_{S i}}{\partial \theta_{3}} \dot{\theta}_{3}, \\
\dot{y}_{S i}=\frac{\partial y_{S i}}{\partial x_{1}} \dot{x}_{1}+\frac{\partial y_{S i}}{\partial \theta_{1}} \dot{\theta}_{1}+\frac{\partial y_{S i}}{\partial \theta_{2}} \dot{\theta}_{2}+\frac{\partial y_{S i}}{\partial \theta_{3}} \dot{\theta}_{3}, \\
\dot{z}_{S i}=\frac{\partial z_{S i}}{\partial x_{1}} \dot{x}_{1}+\frac{\partial z_{S i}}{\partial \theta_{1}} \dot{\theta}_{1}+\frac{\partial z_{S i}}{\partial \theta_{2}} \dot{\theta}_{2}+\frac{\partial z_{S i}}{\partial \theta_{3}} \dot{\theta}_{3},
\end{array}\right.
$$

where $\boldsymbol{\Phi}_{\boldsymbol{i}}$ is the angular displacement of link $i$ in the coordinate system $\left\{O_{3} x_{3} y_{3} z_{3}\right\}$ in Figure $11,\left(x_{S i}, y_{S i}, z_{S i}\right)$ is the centroid position of link $i$.

$$
\tau=H(q) \ddot{q}+C(q, \dot{q}) \dot{q}+G(q),
$$




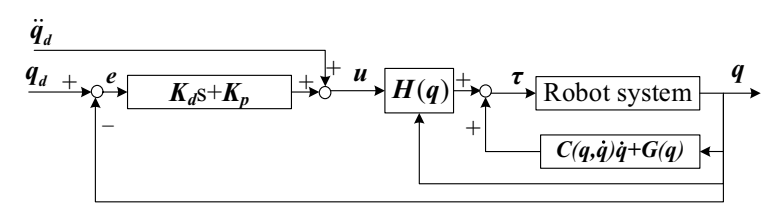

Figure 12 Control block diagram for gravity compensation

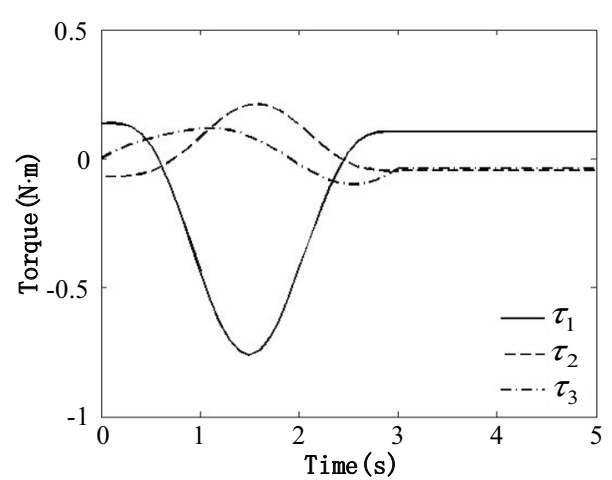

Figure 13 Driving torque variation curves of RCM mechanism

where $\boldsymbol{\tau}$ is the generalized force, $\boldsymbol{H}(\boldsymbol{q}) \ddot{q}$ is the generalized force related with the generalized acceleration, $\boldsymbol{C}(\boldsymbol{q}, \dot{\boldsymbol{q}}) \dot{\boldsymbol{q}}$ is the quadratic form of the generalized velocity, $\boldsymbol{G}(\boldsymbol{q})$ denotes gravity, $\boldsymbol{q}, \dot{\boldsymbol{q}}, \ddot{\boldsymbol{q}}$ are position, velocity and acceleration of the driving joints.

Expression and structure diagram of the controller are shown in Eq. (9) and Figure 12, respectively. The initial position of driving variables is set as $\theta_{1}=44.05^{\circ}$, $\theta_{2}=135^{\circ}, \theta_{3}=0^{\circ}$, and the desired position after $3 \mathrm{~s}$ is $\theta_{1}=40^{\circ}, \theta_{2}=120^{\circ}, \theta_{3}=45^{\circ}$. Thus, the joint trajectory can be described as Eqs. (10), (11), (12) in presupposition. Setting the parameters of the controller as $K_{\mathrm{d}}=\operatorname{diag}(4.5,3.8,8.5)$ and $K_{\mathrm{p}}=\operatorname{diag}(0.0288,0.03888$, 0.26 ), the variation curve of driving torque for each joint is solved as shown in Figure 13. The gravity compensation value of three driving joints can be obtained as $0.1043 \mathrm{Nm},-0.0465 \mathrm{Nm}$ and $0.0361 \mathrm{Nm}$.

$$
\tau=H(q)\left(\ddot{q}_{\mathrm{d}}+K_{\mathrm{d}} \dot{e}+K_{\mathrm{p}} \boldsymbol{e}\right)+\boldsymbol{C}(\boldsymbol{q}, \dot{\boldsymbol{q}}) \dot{\boldsymbol{q}}+\boldsymbol{G}(\boldsymbol{q}),
$$

where $\boldsymbol{e}=\boldsymbol{q}_{\mathrm{d}}-\boldsymbol{q}, \dot{\boldsymbol{e}}=\dot{\boldsymbol{q}}_{\mathrm{d}}-\dot{\boldsymbol{q}}, \boldsymbol{q}_{\mathrm{d}}, \dot{\boldsymbol{q}}_{\mathrm{d}}, \ddot{\boldsymbol{q}}_{\mathrm{d}}$ are desired position, desired velocity and desired acceleration of the driving joints, respectively, and $K_{\mathrm{d}}$ and $K_{\mathrm{p}}$ are PD matrices.

$$
\theta_{1}=\left\{\begin{array}{l}
-1.5 t^{3}+0.75 t^{4}-0.1 t^{5}+44.05 \quad 0<t<3 \\
40 \quad t>3
\end{array}\right.
$$

$$
\begin{aligned}
& \theta_{2}= \begin{cases}-5.56 t^{3}+2.78 t^{4}-0.37 t^{5}+135 & 0<t<3 \\
120 \quad t \geq 3\end{cases} \\
& \theta_{3}= \begin{cases}16.67 t^{3}-8.33 t^{4}+1.11 t^{5} & 0<t<3 \\
45 \quad t \geq 3\end{cases}
\end{aligned}
$$

\subsection{Map Relationship between the Master and the Slave}

The robotic coordinate systems are exhibited in Figure 14. The $z$ directions are same in all the coordinate systems. $\left\{O_{\text {base } 1}\right\}$ and $\left\{O_{\text {base } 2}\right\}$ are the base coordinate systems of the slave manipulators, $\left\{O_{\text {base } 1}^{\prime}\right\}$ and $\left\{O_{\text {base } 2}^{\prime}\right\}$ are the base coordinate systems of the RCM mechanisms, $\left\{O_{s 1}\right\}$ and $\left\{O_{s 2}\right\}$ are the end-effector coordinate systems where the $x$ direction is same with the base coordinate systems of the RCM mechanisms. To achieve the surgeon's intuitive operation, the coordinate systems of the left robot (including the left slave manipulator and the left master controller) are established based on the Left-hand rule while the right ones are established based on the Righthand rule. Furthermore, the coordinate systems of the end-effectors under the view of the microscope match with the ones of the master controllers to surgeon. The motion increment of the master controller is represented as $\Delta \overrightarrow{\boldsymbol{d}}_{\mathrm{m}}$; thus, the motion increment of the end-effector can be calculated as $\Delta \overrightarrow{\boldsymbol{d}}_{\mathrm{s}}=k_{\mathrm{m}} \Delta \overrightarrow{\boldsymbol{d}}_{\mathrm{m}}$, where $k_{\mathrm{m}}$ is the scale factor usually less than 1 . Then the desired position of the end-effector after the incremental motion can be derived as $\overrightarrow{\boldsymbol{d}}_{s}=\Delta \overrightarrow{\boldsymbol{d}}_{s}+\overrightarrow{\boldsymbol{d}}_{s-1}$, where $\overrightarrow{\boldsymbol{d}}_{s-1}$ is the previous position. Finally, the joint variables of the slave manipulator can be obtained by the inverse kinematics described in Section 4.2.

\subsection{Tremor Cancelling}

It is necessary to cancel the surgeon's hand tremor during the operation since the tremor could influence surgical treatment in retinal surgery. The frequency of hand tremor is largely in the range from 2.5 to $12.5 \mathrm{~Hz}$, and signal to noise ratio (SNR) of the tremor signal is 0.93 compared to the useful signal [35]. In this work, recursive least-squares (RLS) adaptive algorithm [36] is applied to recover the input operation signal from the disturbed signal blended with the tremor signal. The tremor signal $n(t)$ is defined by the Gaussian white noise with $0.93 \mathrm{SNR}$, and the desired input signal is defined as 


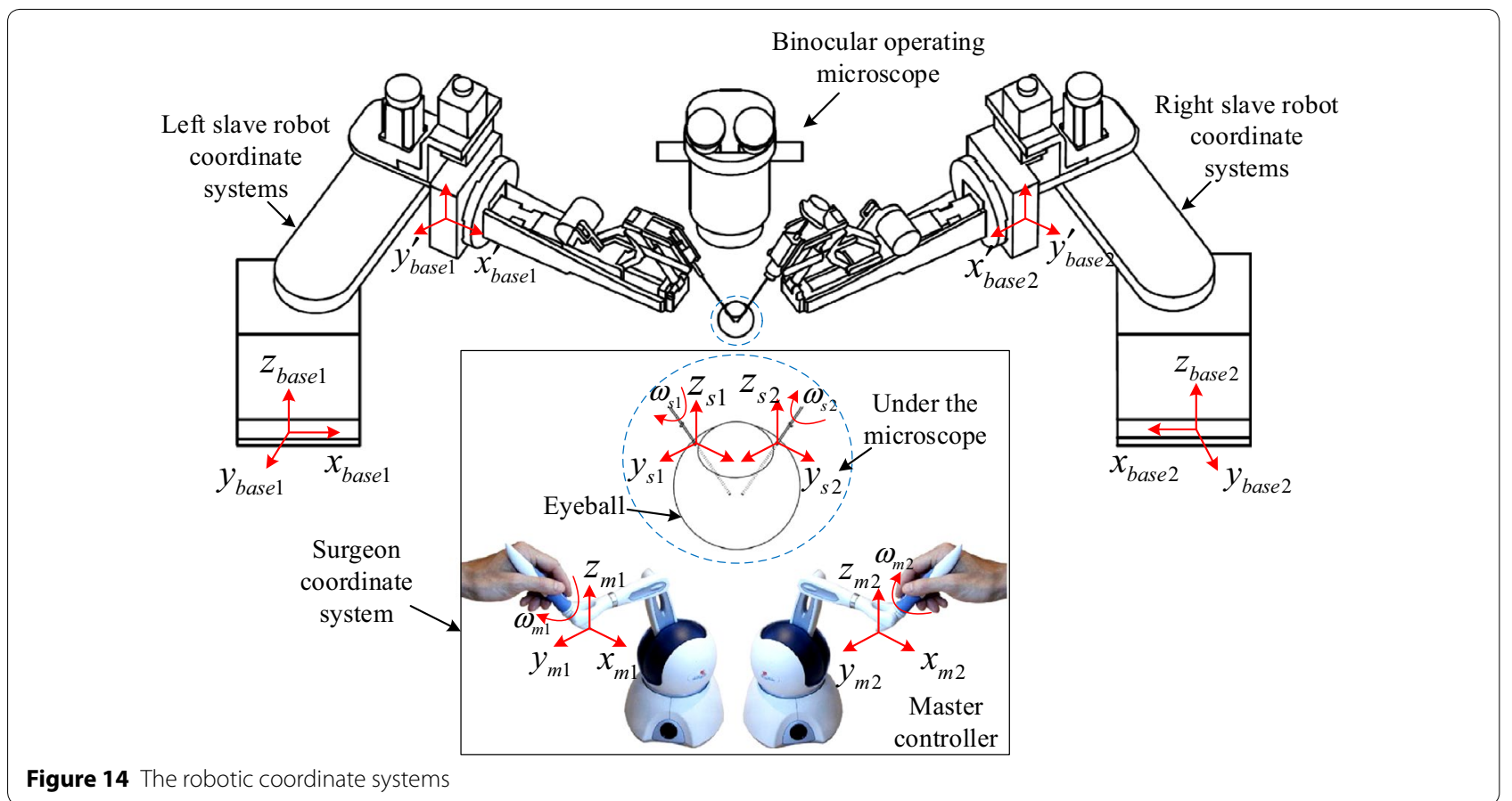

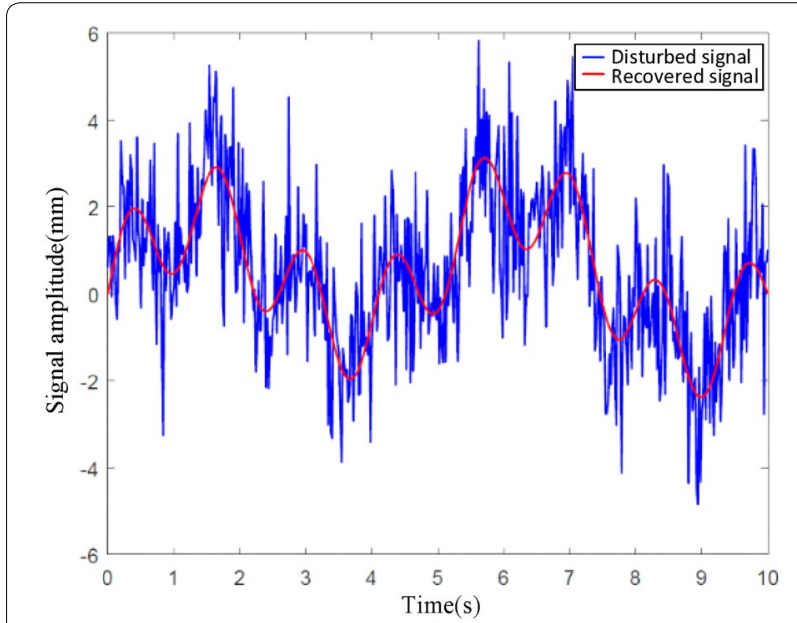

Figure 15 Comparison between the disturbed signal and the signal recovered by $R L S$ algorithm

$$
d(t)=0.5 \sin (0.4 \pi t)+0.1 \sin (0.1 \pi t)+0.8 \sin (1.5 \pi t) .
$$

The desired signal $d(t)$ is involved in the tremor signal $n(t)$, thus the actual input signal $s(t)$ can be written as

$$
s(t)=d(t)+n(t) .
$$

The desired input signal is successfully recovered from the disturbed signal as shown in Figure 15, where the relative error is less than $9.7334 \times 10^{-5}$.

\section{Experiments and Results}

The experiments on an artificial eye model (Figure 16) and an in vitro porcine eye (Figure 17) are conducted to examine the feasibility of the robotic system and the operation process. The artificial eye model is made of a plastic ball of $24 \mathrm{~mm}$ diameter (same with the human eye), and two trocars are plugged in the two sides of the model. Due to its simple structure, the artificial eye model is only used in the preliminary experiments. In the contrast, the in vitro porcine eyeball is treated as the real experimental target and the whole operation process is tested. The porcine eyeball is provided by the slaughter house within four hours after the porcin being slaughtered. During the experiment, the porcine eye is supported by a custom-designed PMMA stand which is also used for position calibration.

The experiment on the porcine eyeball is conducted in several steps. The first step "translation" is implemented to locate the sclerotomy site and align the instrument tip point with the sclerotomy site. In this mode, only the PC host computer is active to control the robot, and only the SCARA stages are actuated during this mode. The next step "pose adjustment" is applied to obtain a suitable pose of the instrument for further passing through the trocar, the robot is still controlled only by the PC host computer in this step, and the movements of the RCM mechanisms contribute to the tilt and roll pose adjustment of the end-effector. Then, the robotic system in the following steps is manipulated by the main surgeon with 


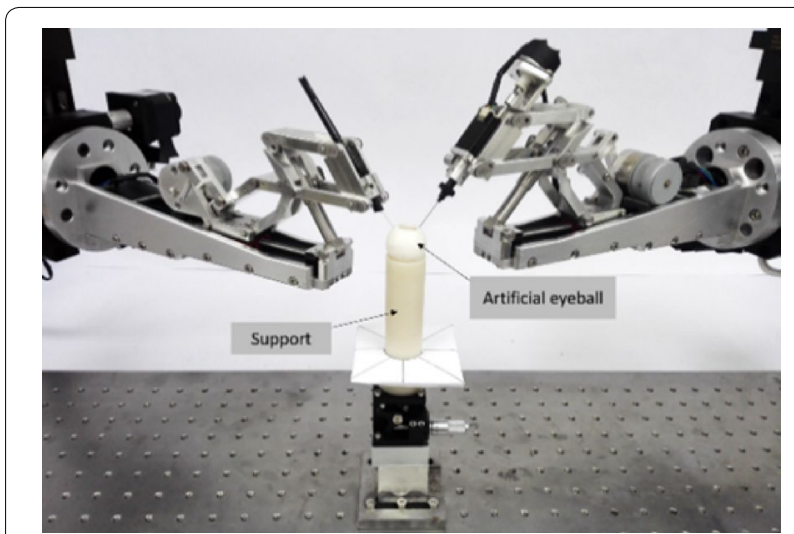

Figure 16 Experiments on an artificial eye model

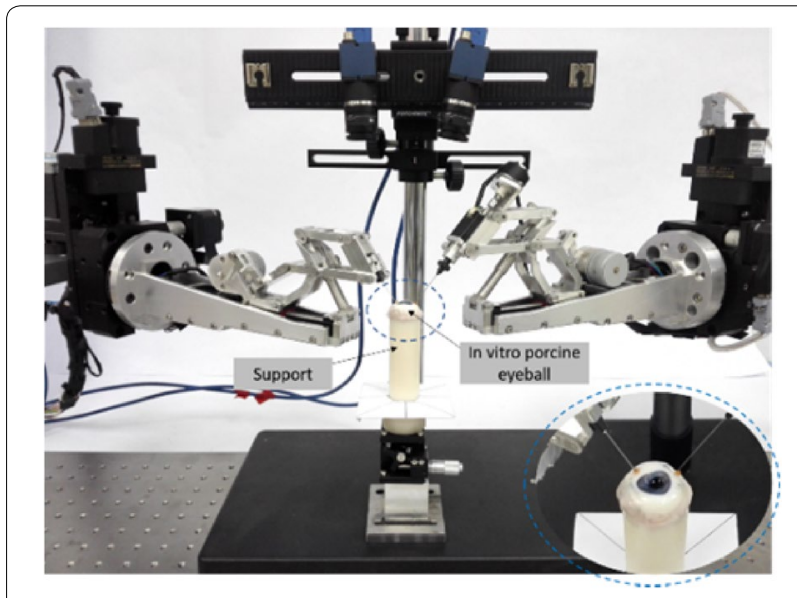

Figure 17 Experiments on an in vitro porcine eye

the master controller. Firstly, the step "insertion" is conducted to insert the instrument into the eye model, the instrument is moved into the eye model along its axial direction consecutively or intermittently without damage to the scleral insertion point. Then, the instrument tip is moved to a desired location and touches the target point on the retina to accomplish particular tasks such as stenting or endodiathermy. When the surgical operation is completed, the "retreat" mode is activated to remove the instrument from the eyeball by pressing the exit button on the master controller. Finally, the robot joints return to the initial site in "return-to-zero" mode controlled by the PC host computer. The experiments have demonstrated the possibility of the clinical use of the robot assisted RVBS in the future.

\section{Conclusions}

1. This paper focuses on the research and the realization of a robotic system assisted for RVBS, and this is also the early development of the ophthalmic robotic system in China.

2. The system consists of two salve manipulators and two master controllers. The slave manipulator is made up of a novel RCM mechanism (three DoF), a SCARA stage (three DoF), a novel end-effector (two DoF), and PHANTOM Omni as the master controller.

3. Control strategies, including motion scaling, gravity compensation and tremor canceling, are applied in the robotic system to achieve the precise control.

4. In the preliminary experiments, feasibility of the system is validated. The whole surgery operation procedures are conducted assisted by the robotic system and the system shows a good performance.

5. The novelty of this work includes: (a) a novel RCM mechanism is applied as the slave manipulator in the robot, this RCM mechanism enables robot to spatially rotate the instrument around a virtual fixed point as well as insert or retract the tool, (b) a novel end-effector is designed and enables robot to open or close the microforceps jaw, and rotate the microforceps around its axis, (c) the gravity compensation result and hand tremor filter provides steady motion of the robot, improves the manipulation safety.

6. In future, the force sensor will be integrated in surgical tool and the vision-based localization for endeffector will be implemented. In addition, more experiments will be carried out to quantify the differences between manual and robot-assisted procedures in RVBS.

\section{Authors' Contributions}

$\mathrm{C}-\mathrm{YH}$ was in charge of the whole trial, design and implemented the control systems, end-effector, kinematic analysis, and wrote the manuscript; LH designed RCM mechanism; YY provides workflow of the paper and experiment; Q-FL assisted to conduct the experiments; Y-KL assisted to analyze the dynamic systems. All authors read and approved the final manuscript.

\section{Author details}

${ }^{1}$ School of Mechanical Engineering and Automation, Beihang University, Beijing 100191, China. ${ }^{2}$ Beijing Institute of Ophthalmology, Beijing Tongren Eye Center, Beijing Tongren Hospital, Capital Medical University, Beijing Key Laboratory of Ophthalmology and Visual Sciences, Beijing 100005, China.

\section{Authors' Information}

Chang-Yan He, born in 1993, is currently a PhD candidate at School of Mechanical Engineering and Automation, Beihang University, China. He received his bachelor degree from Beijing Jiaotong University, China, in 2015. His research focuses on medical robotics.

Long Huang, born in 1988, is currently a PhD candidate at School of Mechanical Engineering and Automation, Beihang University, China. He received his master degree from School of Mechanical Engineering and Automation, Beihang University, China. His research interests include mechanism and robotics.

Yang Yang, born in 1962, is currently a professor and a PhD candidate supervisor at School of Mechanical Engineering and Automation, Beihang 
University, China. His main research interests include mechachonics engineering, robotics, and multi-fingered dexterous hand.

Qing-Feng Liang, born in 1971, is currently an associate chief physician and a professor at Beijing Institute of Ophthalmology, Beijing Tongren Eye Center, Beijing Tongren Hospital, Capital Medical University, Beijing Key Laboratory of Ophthalmology and Visual Sciences, Beijing.

Yong-Kang Li, born in 1985, is currently a PhD candidate at School of Mechanical Engineering and Automation, Beihang University, China. His research interests include mechanism and robotics.

\section{Competing Interests}

The authors declare that they have no competing interests.

\section{Funding}

Supported by National Natural Science Foundation of China (Grant Nos. 50675008, 51175013), National Hi-tech Research and Development Program of China (863 Program, Grant No. 2017YFB1302702).

\section{Publisher's Note}

Springer Nature remains neutral with regard to jurisdictional claims in published maps and institutional affiliations.

Received: 6 June 2017 Accepted: 16 August 2018

Published online: 28 August 2018

\section{References}

[1] A Sharma, D J D'amico. Medical and surgical management of central retinal vein occlusion. International Ophthalmology Clinics, 2004, 44(1): $1-16$.

[2] S Rogers, R L Mclntosh, N Cheung, et al. The prevalence of retinal vein occlusion: pooled data from population studies from the United States, Europe, Asia, and Australia. Ophthalmology, 2010, 117(2): 313-319.

[3] C N Riviere, P S Jensen. A study of instrument motion in retinal microsurgery. Proceedings of the 22nd Annual International Conference of the IEEE Engineering in Medicine and Biology Society, Chicago, USA, July 23-28, 2000: 59-60.

[4] J J Xiao, Y Yang, D Z Li, et al. Advances and key techniques of ophthalmic microsurgical robots. Journal of Mechanical Engineering, 2013, 49(1): 15-22. (in Chinese)

[5] A Guerrouad, P Vidal. S.M.O.S.: Stereotaxical microtele- manipulator for ocular surgery. Proceedings of the Annual International Conference of the IEEE Engineering in Medicine and Biology Society, Seattle, USA, November 9-12, 1989: 879-880.

[6] DY Yu, S J Cringle, I J Constable. Robotic ocular ultramicrosurgery. Australian and New Zealand Journal of Ophthalmology, 1998, 26(Suppl.): 6-8.

[7] S Charles, H Das, TOhm, et al. Dexterity-enhanced telerobotic microsurgery. 8th International Conference on Advanced Robotics, Monterey, USA, July 7-9, 1997: 5-10.

[8] W Wei, R Goldman, N Simaan, et al. Design and theoretical evaluation of micro-surgical manipulators for orbital manipulation and intraocular dexterity. 2007 IEEE International Conference on Robotics and Automation, Roma, Italy, April 10-14, 2007: 3389-3395.

[9] T Ueta, Y Yamaguchi, Y Shirakawa, et al. Robot-assisted vitreoretinal surgery: development of a prototype and feasibility studies in an animal model. Ophthalmology, 2009, 116(8): 1538-1543.

[10] A Üneri, M A Balicki, J Handa et al. New steady-hand eye robot with micro-force sensing for vitreoretinal surgery. 3rd IEEE RAS and EMBS International Conference on in Biomedical Robotics and Bio Mechatronics, Tokyo, Japan, September, 2010: 814-819.

[11] E Rahimy, J Wilson, T Tsao, et al. Robot-assisted intraocular surgery: development of the iriss and feasibility studies in an animal model. Eye, 2013, 27(8): 972.

[12] M A Nasseri, M Eder, S Nair, et al. The introduction of a new robot for assistance in ophthalmic surgery. 35th Annual International Conference of the IEEE Engineering in Medicine and Biology Society, Osaka, Japan, July 3-7, 2013: 5682-5685.
[13] A Gijbels, E B Vander Poorten, P Stalmans, et al. Design of a teleoperated robotic system for retinal surgery. 2014 IEEE International Conference on Robotics and Automation, Hong Kong, China, May 31-June 7, 2014: 2357-2363.

[14] M D Smet, T C Meenink, T Janssens, et al. Robotic assisted cannulation of occluded retinal veins. Plos One, 2016, 11 (9): e0162037.

[15] P Su, Y Yang, Y Song. Corneal hyper-viscoelastic model: derivations, experiments, and simulations. Acta of Bioengineering and Biomechanics, 2015, 17(2): 73-84

[16] Y Yang, C L Xu, S S Deng, et al. Insertion force in manual and robotic corneal suturing. The International Journal of Medical Robotics and Computer Assisted Surgery, 2012, 8(1): 25-33.

[17] P Su, Y Yang, L Huang. Biomechanical simulation of needle insertion into cornea based on distortion energy failure criterion. Acta of Bioengineering and Biomechanics, 2016, 18(1): 25-33.

[18] J J Xiao, L Huang, L J Shen, et al. Design and research of a robotic aided system for retinal vascular bypass surgery. ASME Journal of Medical Devices, 2014, 8(4): 044501-1-044501-6.

[19] J L Bourges, J P Hubschman, J Wilson, et al. Assessment of a hexapod surgical system for robotic micro-macro manipulations in ocular surgery. Ophthalmic Research, 2011, 46(1): 25-30.

[20] R A MacLachlan, B C Becker, J C Tabar'es, et al. Micron: an actively stabilized handheld tool for microsurgery. IEEE Transactions on Robotics, 2012, 28(1): 195-212.

[21] B Gonenc, M A Balicki, J Handa, et al. Preliminary evaluation of a microforce sensing handheld robot for vitreoretinal surgery. Proceedings of IEEE/ RSJ International Conference of Intelligent Robots and Systems, Algarve, Portugal, October 7-12, 2012: 4125-4130.

[22] J Hubschman, J Bourges, W Choi, et al. The micro hand: a new concept of micro-forceps for ocular robotic surgery. Eye, 2010, 24(2): 364.

[23] X He, V Van Geirt, P Gehlbach, et al. Iris: Integrated robotic intraocular snake. Proceedings of IEEE International Conference of Robotics and Automation, May 26-30, Seattle, Washington, USA, 2015: 1764-1769.

[24] M P Kummer, J J Abbott, B E Kratochvil, et al. Octomag: an electromagnetic system for 5-dof wireless micromanipulation. IEEE Transactions on Robotics, 2010, 26(6): 1006-1017.

[25] X He, J Handa, P Gehlbach, et al. A submillimetric 3-DoF force sensing instrument with integrated fiber bragg grating for retinal microsurgery. IEEE Transactions on Biomedical Engineering, 2014, 61(2): 522-534.

[26] X He, M Balicki, P Gehlbach, et al. A multi-function force sensing instrument for variable admittance robot control in retinal microsurgery. IEEE International Conference of Robotics and Automation, Hong Kong, China, May 31-June 5, 2014: 1411-1418.

[27] F Walsh. Robot operates inside eye in world first, 2016. [Online]. Available: http://www.bbc.com/news/health-37246995.

[28] T Donald. Robot assists with injections for rvo. Retina Today, 2017, 4: 59-61.

[29] N Berker, C Batman. Surgical treatment of central retinal vein occlusion. Acta Ophthalmologic, 2008, 86(3): 245-252.

[30] L J Shen, Y Q Chen, L L Wei, et al. Bypassing occluded retinal main vessel segments in isolated arterially perfused caprine eyes. Current Eye Research, 2009, 34(6): 415-420.

[31] L J Shen, Y Q Chen, D Cheng, et al. In vivo retinal vein bypass surgery in a porcine model. Current Eye Research, 2016, 41(1): 79-87.

[32] L Huang, Y Yang, J J Xiao, et al. Type synthesis of 1R1T remote center of motion mechanisms based on pantograph mechanisms. Journal of Mechanical Design, 2016, 138(1): 014501.

[33] J J Craig. Introduction to robotics: mechanics and control. Boston: AddisonWesley Pub. Co., 2005.

[34] W Huo. Robot dynamics and control. Beijing: Higher Education Press, 2005. (in Chinese)

[35] J Timmer. Modeling noisy time series: physiological tremor. International Journal of Bifurcation and Chaos, 1998, 8(7): 1505-1516.

[36] C Zhao. Adaptive signal processing technology. Beijing: Beijing Institute of Technology Press, 2009. (in Chinese) 